\title{
Two Stage Multi Sensor Detection Scheme for Large Scale WSN Using Spatial Proximity
}

\author{
Sadiq Ali ${ }^{1}$, Feroz Shah' ${ }^{2}$ Eid Badshah ${ }^{3}$ \\ RECEIVED ON 25.12.2019, ACCEPTED ON 15.09.2020
}

\begin{abstract}
Intuition suggests that in a large-size sensor network, event signal spreads over a sub-region known as event region. The sub-set of sensors inside the event region is located close to each other in the form of a cluster. On the contrary, the reports from the rest of the sensors that are far away from the event will be less credible and prone to faulty detection. In this work, such fact is exploited to improve the detection performance of the detection schemes at the fusion center by proposing a two-stage detection mechanism. In other words, the proposed two-stage detection scheme exploits the spatial proximity of the event region sensors. Numerical results are provided to assess the detection performance of the proposed scheme that shows that the proposed detection scheme has a superior performance over the traditional detection schemes.
\end{abstract}

Keywords: Wireless Sensor Network, GLRT, Spatial Proximity, Event Region, Detection

\section{INTRODUCTION}

A network of very small, low-cost, low-power, and autonomous sensor nodes is known as a Wireless Sensor Network (WSN). The task of these nodes is to sense the environment by gathering the information at the sensors from the surrounding environment and collaboratively transfer the gathered information to the fusion center for further processing [1-5]. In the sensing process of WSNs, detection is the most important and an initial step [6, 7]. For example, in the monitoring of an environment, the presence of contaminant (e.g., radioactive material) is detected first before finding the level of contamination [8]. Mostly in large scale WSNs, the signal level produced by such events/targets/contamination may spread over a portion of the whole region, which is known as the event region $[8,9]$. In other words, the event region spreads over an area that includes just a subset of all the sensor nodes. On the contrary, the far-away sensors are typically unable to receive the event signals as the sensors will have limited sensing ranges. Furthermore, the sensors located closer to the event (inside the event region) will often be forming a cluster and the observations of these sensors will be highly correlated $[9,10]$.

Most of the current detection techniques are simply based on energy detection. The importance of the cross-sensor correlation information between these closely located sensors (sensor located inside the event region) is ignored [11-13]. The structure present within the received samples at the subset of sensors can be used to improve the detection performance further. For example, taking into account such side information, we can address issues related to data fusion, reliability of the information from the sensors and fault detection $[6,7,14]$. Therefore, the main goal

\footnotetext{
${ }^{1}$ Department of Electrical Engineering, University of Engineering and Technology, Peshawar, Pakistan.

Email:sadiqali@uetpeshawar.edu.pk

${ }^{2}$ Department of Mechanical Engineering, University of Engineering and Technology, Peshawar, Pakistan. Email: ferozshah@uetpeshawar.edu.pk

${ }^{3}$ Department of Civil Engineering, University of Engineering and Technology, Peshawar, Pakistan.

Email: eid_shah@uetpeshawar.edu.pk
}

This is an open access article published by Mehran University of Engineering and Technology, Jamshoro under CC BY 4.0 International License. 
of this work is to design another decision fusion rule that adds robustness against the faulty detection through (1) selection of the important sensors and (2) the spatial proximity among these important sensors.

The problem of selecting a subset of sensors has been previously addressed in [15], where a technique for signal detection is proposed that identifies the useful observations among the set of all received measurements from local sensors. The selection process is formulated in terms of the Multifamily Likelihood Ratio Test (MFLRT) that jointly performs the detection and selection using model order selection criterion [15]. This method outperforms the state of the art methods such as the Generalized Likelihood Ratio Test (GLRT) which is asymptotically optimal. In our earlier work [16, 17], we proposed detection schemes that exploit both the selection of the useful set of samples (order to reject noise) and advantage of the signal correlation due to the presence of the emitting target. In these papers, signal correlation is exploited using the concept of "spatial signatures", where a spatial signature has been adopted as a way to capture the structure of spatially correlated measurements of neighboring sensor nodes. It is shown that the proposed approaches significantly improve the detection performance compared to the traditional GLRT approach. However, in these methods, all sensors send information unnecessary to the fusion center that results in high communication costs and thus low power efficiency. Similarly, MFLRT is an iterative method that results in huge computational cost in exploiting spatial structure by processing data of the whole sensor network.

In order to overcome such shortcomings of our earlier work, in the proposed decision rule/scheme the following steps are devised:

1. With the help of local decisions at each sensor, Local censoring is performed and then based on these local decisions only a subset of sensors transmits its information to the fusion center thus avoiding unnecessary communication costs.

2. The proposed scheme exploits spatial proximity in a simple and cost-effective way to reduce the computational cost at the fusion center by exploiting the available side information of the position of the sensors.

Mehran University Research Journal of Engineering and Technology, Vol. 41, No. 1, January 2022 [p-ISSN: 0254-7821, e-ISSN: 2413-7219]
At the end of this work, it will be shown that this approach significantly improves the detection performance compared to traditional suboptimal approaches such as the GLRT [15]. We further remark that the proposed scheme can be equally effective in identification and isolation of unreliable sensor nodes and malicious nodes.

\section{SYSTEM MODEL}

We consider $\mathrm{K}$ sensor nodes spread over a large area $\mathcal{A} \subset \mathbb{R}^{2}$, where the coordinate of the $\mathrm{k}$-th sensor are $\left(\mathrm{a}_{\mathrm{k}}, \mathrm{b}_{\mathrm{k}}\right)$ for $\mathrm{k}=1,2, \ldots, \mathrm{K}$, as illustrated in Fig. 1 for $\mathrm{K}=5$ sensors. The coordinates of the sensors are considered to be known to the fusion center as we could easily assume that locations of the sensor nodes are a priori known or the sensor nodes are GPSenabled [18, 19]. The task of these nodes is to monitor the environment and detect the presence of an event at unknown random location. Let us assume that the unknown coordinates of the event are $\left(a_{t}, b_{t}\right)$. At the $\mathrm{k}$-th sensor, $\mathrm{k}=1,2, \cdots, \mathrm{K}$, the signal model for the received signals under the alternative hypotheses can be represented as:

$\mathcal{H}_{0}: \mathrm{y}_{\mathrm{k}}(\mathrm{n})=\mathrm{v}_{\mathrm{k}}(\mathrm{n}) \quad \mathcal{H}_{1}: \mathrm{y}_{\mathrm{k}}(\mathrm{n})=\eta_{\mathrm{k}}+v_{k}(\mathrm{n})$

where $\mathrm{n}=1,2, \cdots, \mathrm{N}, \eta_{\mathrm{k}}=\sqrt{\mathrm{P}_{\mathrm{k}}}$ and $\mathrm{P}_{\mathrm{k}}=\mathrm{P}_{0} /(1+$ $\alpha \mathrm{d}_{\mathrm{k}}^{\beta}$ ) is the unknown received power at the $\mathrm{k}$-th sensor. Whereas, $P_{0}$ is emitted power at the event/target, parameter $\beta, 2 \leq \beta \leq 5$ is known as a signal decaying exponent, and $\mathrm{d}_{\mathrm{k}}=$ $\sqrt{\left(a_{k}-a_{t}\right)^{2}+\left(b_{k}-b_{t}\right)^{2}}$ is the euclidean distance between the $\mathrm{k}$-th sensor and target/event, which is considered to be unknown. The adjustable parameter is denoted by $\alpha$, as larger $\alpha$ means signal power is decaying faster [20]. Similarly, $\mathrm{v}_{\mathrm{k}}(\mathrm{n}) \sim \mathcal{N}\left(0, \sigma_{\mathrm{v}}^{2}\right)$ is Additive White Gaussian Noise (AWGN) that is independent identically distributed. The signal model under consideration can be described for acoustic and electromagnetic signals [21].

The local sensors take observations of the environment; they send their observations to the central unit known as the fusion center. The remaining processing of the signal is performed at the fusion center to make the final decision. 


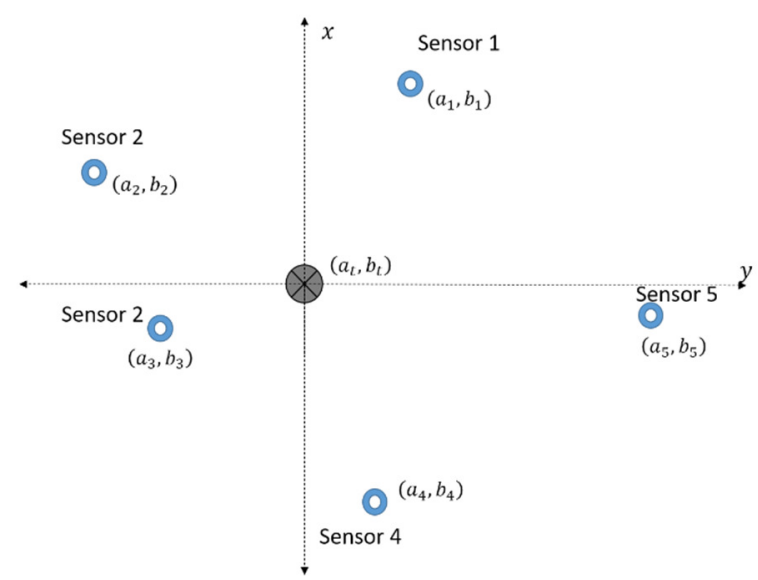

Fig.1: Illustration of the sensor field in 2 dimensions

\section{PROPOSED TWO STAGE DETECTION SCHEME}

The main goal of this work is to design a fusion rule that takes into account the importance of those sensors that provide more credible reports to the fusion center by exploiting spatial proximity. With this goal, the proposed detection mechanism is illustrated in Fig. 2. The proposed scheme is performed in two stages.

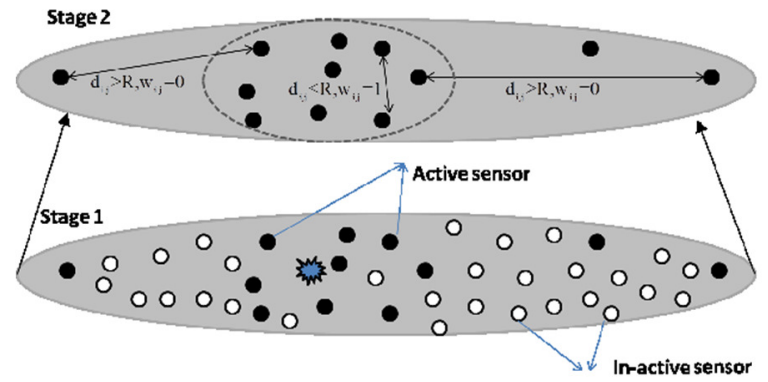

Fig. 2: Illustration of the two stage detection mechanism

Stage 1: Local classification of K sensors being either credible and non-credible.

Stage 2: Fusion level confirmation of the credible sensors by using their proximity.

\subsection{Stage 1: Local Decisions}

In this section, we present the first stage of the two stage scheme, where detector at local sensors is used to decide whether it is in any of the following two categories:

1. Active Sensors: These sensor are the one that are supposed to be inside the event region and their observations are credible.

2. Non-Active Sensors: These sensors are supposed to be far away from the event region and their observations are considered to be less credible.

For making such decision, the GLRT is used at each local nodes [17]. For calculating the GLRT based detector, $\mathrm{k}$-th sensor $(\mathrm{k}=1,2, \cdots, \mathrm{K})$ takes $\mathrm{N}$ samples. Lets $\mathbf{y}_{\mathrm{k}} \triangleq\left[\begin{array}{llll}\mathrm{y}_{\mathrm{k}}(1) & \mathrm{y}_{\mathrm{k}}(2) & \cdots & \mathrm{y}_{\mathrm{k}}(\mathrm{N})\end{array}\right]^{\mathrm{T}}$ is a vector that contains $\mathrm{N}$ received samples at the $\mathrm{k}$-th sensor. Now the k-th sensor makes the local decision by comparing the GLRT test statistic with a threshold. For the case of known noise power, the GLRT statistic can be expressed as:

$\mathrm{L}_{\mathrm{G}}\left(\mathbf{y}_{\mathrm{k}}\right)=\frac{\operatorname{maxf}_{\eta_{\mathrm{k}}}\left(\mathbf{y} ; \eta_{\mathrm{k}}, \mathcal{H}_{1}\right)}{\mathrm{f}\left(\mathbf{y} ; \mathcal{H}_{0}\right)}$

where $\quad \mathrm{f}\left(\mathbf{y} ; \eta_{\mathrm{k}}, \mathcal{H}_{1}\right)=\Psi \exp \left[-\frac{1}{2 \sigma_{\mathrm{v}}^{2}} \sum_{\mathrm{n}=1}^{\mathrm{N}}\left(\mathrm{y}_{\mathrm{k}}(\mathrm{n})-\right.\right.$ $\left.\left.\eta_{\mathrm{k}}\right)^{2}\right]$ with $\Psi=\left(2 \pi \sigma_{v}^{2}\right)^{-\frac{1}{2}}$ is the probability distribution of the received signals under hypothesis $\mathcal{H}_{1}$ and $\mathrm{f}\left(\mathbf{y} ; \mathcal{H}_{0}\right)=\Psi \exp \left[-\frac{1}{2 \sigma_{\mathrm{v}}^{2}} \sum_{\mathrm{n}=1}^{\mathrm{N}} \mathrm{y}_{\mathrm{k}}^{2}(\mathrm{n})\right]$ is the probability distribution of the received signals under hypothesis $\mathcal{H}_{0}$. Solving ((2)), we can get the GLRT based local detector as [17]:

$\Lambda_{\mathrm{k}}=2 \ln L_{\mathrm{G}}\left(\mathbf{y}_{\mathrm{k}}\right)=\frac{\mathrm{N}_{\mathrm{k}}^{2}}{\sigma_{\mathrm{v}}^{2}} \underset{\text { NonActive }}{\stackrel{\text { Active }}{\gtrless}} \gamma$

where $\gamma$ is known as a threshold and $\bar{y}_{\mathrm{k}}=$ $\frac{1}{2} \sum_{n=1}^{N} y_{k}(n)$. For the implementation of the (2), local threshold is required to be known. For this probability of false alarm $\mathrm{P}_{\mathrm{FA}}$ should be known. For $\mathrm{P}_{\mathrm{FA}}$, the Probability Distribution Function (PDF) of the term $\Lambda_{\mathrm{k}}$ is required. The PDF of test statistic (2) can be expressed by the Chi-Square distribution with 1 degree of freedom as [17]:

$\mathcal{H}_{0}: \Lambda_{\mathrm{k}} \sim \chi_{1}^{2} \quad \mathcal{H}_{1}: \Lambda_{\mathrm{k}} \sim \chi_{1}^{2}\left(\frac{\mathrm{N} \eta_{\mathrm{k}}^{2}}{\sigma_{\mathrm{v}}^{2}}\right)$

Now as we have the PDF of $\Lambda_{\mathrm{k}}$, using ((4)) $\mathrm{P}_{\mathrm{FA}}$ is $\mathrm{P}_{\mathrm{FA}}=\mathrm{Q}_{\chi_{1}^{2}}(\gamma)$, where $\mathrm{Q}_{\chi_{1}^{2}}$ is right-tail probability of $\chi_{1}^{2}$ [22]. The threshold $\gamma$ can be calculated as $: \gamma=$ $\mathrm{Q}_{\chi_{1}^{2}}^{-1}\left(\mathrm{P}_{\mathrm{FA}}\right)$, which is then utilized to find $((2))$. We remark that $((2))$ is used at every sensor find the 
decision. The local decision helps us to know whether the local sensors are non-active or active. Where active sensors are those which decide that event is present. Such local information is then used to find the proposed fusion roles at the fusion center.

\subsection{Stage two: Proximity-Based Event Confirmation}

This section presents stage two of the proposed scheme when selected local sensors ( $L$ active sensors) send soft information (i.e., $l$-th sensor sends $\bar{y}_{\mathrm{k}}=$ $\left.\frac{1}{\mathrm{~N}} \sum_{\mathrm{n}=1}^{\mathrm{N}} \mathrm{y}_{\mathrm{k}}(\mathrm{n})\right)$ to the fusion center. Now at the fusion center, we have two type information:

1. $\mathbf{x}=\left[\overline{\mathrm{y}}_{1}, \overline{\mathrm{y}}_{2}, \ldots, \overline{\mathrm{y}}_{\mathrm{L}}\right]$

2. Information about the location of the $L$ active sensors.

In order to design the fusion rule, we exploit the above information to make the final decision. In other words, for confirmation of the local decision, proximity information of the active sensors is exploited to perform the detection mechanism. With this approach, we can also detect the unreliable sensor nodes and malicious sensor nodes [23]. In order to do so we adopt the quadratic form [15, 24]:

$$
\Lambda(\mathbf{x})=\mathbf{x}^{\mathrm{T}} \mathbf{W} \mathbf{\mathbf { H } _ { 1 }} \underset{\mathcal{H}}{\gtrless} \gamma_{\mathrm{F}}
$$

The statistical dependency amongst the neighboring active sensors is checked by the weight matrix $\mathbf{W}$, that is a symmetric binary matrix of spatial weights with $w_{i, j}$ is the $(i, j)-$ th element as:

$\mathbf{W}=\left[\begin{array}{llll}w_{1,1} & w_{1,2} & \cdots & w_{1, L} \\ w_{2,1} & w_{2,2} & \cdots & w_{2, L} \\ \vdots & \vdots & \ddots & \vdots \\ w_{L, 1} & w_{L, 2} & \cdots & w_{L, L}\end{array}\right]$

Based on the known location of the $\mathrm{L}$ active sensors, in order to find the correlation weights $w_{i, j}$, we need to construct the mathematical model using geometry given in Fig. 3. In Fig. 3, $\alpha_{i j}$ is the effective angle. Let

$d_{i, j}=\left\|s_{i}-s_{j}\right\|=\sqrt{\left(a_{i}-a_{j}\right)^{2}+\left(b_{i}-b_{j}\right)^{2}}$ is the distance between the two sensors located at the locations $r_{i}=\left(a_{i}, b_{i}\right)$ and $r_{j}=\left(a_{j}, b_{j}\right)$, respectively. We assume coverage to be a Boolean disk model. Let
$R_{i}$ is the circular sensing region of $i$-th sensor with radius $r$ and centered at $r_{i}$. Similarly, $R_{j}$ is the circular sensing region of $j$-th sensor with radius $r$ and centered at $r_{j}$. The fraction of common sensing area covered by the two circular regions represents the correlation weights that can be represented as [25]:

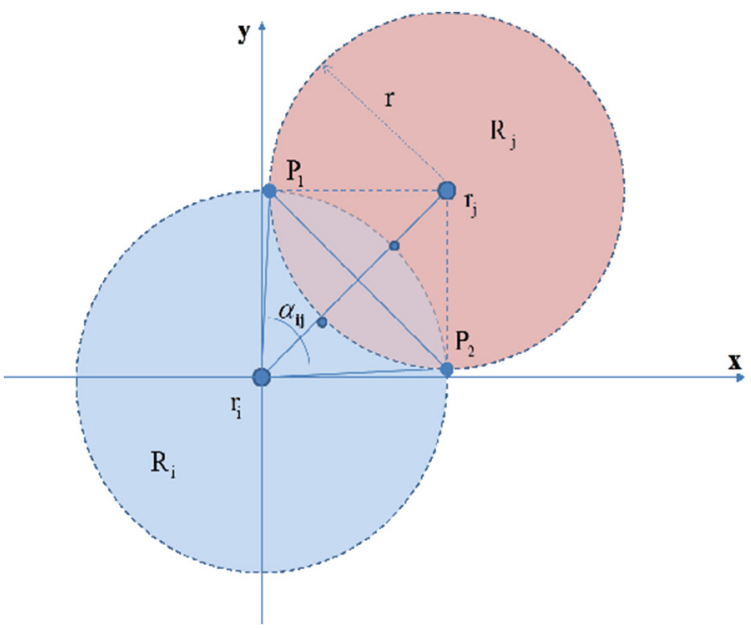

Fig. 3: Spatial Circular Correlation Model [25]

$\mathrm{w}_{\mathrm{i}, \mathrm{j}}=\mathrm{D}_{\theta}\left\{\mathrm{d}_{\mathrm{i}, \mathrm{j}}\right\}=\mathrm{D}_{\theta}\left\{\left\|\mathrm{s}_{\mathrm{i}}-\mathrm{s}_{\mathrm{j}}\right\|\right\}$

We assume all sensors have same sized sensing region. Let $h_{i, j}$ is the chord length that connects the two intersections $\mathrm{P}_{1}$ and $\mathrm{P}_{2}$, and can be represented as [25]:

$h_{i, j}=2 \sqrt{r^{2}-\frac{d_{i j}^{2}}{4}}$

Let $A=\pi r^{2}$ is the region of circular region, $A_{i}^{j}$ is the area of shaded region covered by the the $\overline{\operatorname{arc}} \widehat{\mathrm{P}_{1} \mathrm{P}_{2}}$ for $S_{i}$ and chord $\overline{\mathrm{P}_{1} \mathrm{P}_{2}}$. Similarly, $A_{j}^{i}$ is the area covered by the $\overrightarrow{\operatorname{arc}} \widehat{\mathrm{P}_{1} \mathrm{P}_{2}}$ for $\mathrm{S}_{\mathrm{j}}$ and chord $\overline{\mathrm{P}_{1} \mathrm{P}_{2}}$. Then we can write [25]:

$\mathrm{w}_{\mathrm{i}, \mathrm{j}}=\mathrm{D}_{\theta}\left\{\mathrm{d}_{\mathrm{i}, \mathrm{j}}\right\}=\frac{\mathrm{A}_{\mathrm{i}}^{\mathrm{j}}+\mathrm{A}_{\mathrm{j}}^{\mathrm{i}}}{\mathrm{A}}$

Due to symmetry $A_{i}^{j}=A_{j}^{i}$ as shown in Fig. 2, we can write

$A_{i}^{j}=\frac{r^{2} \cos ^{-1}\left(\frac{d_{i, j}}{2 r}\right)}{2}-\frac{h_{i, j} d_{i j}}{4}=A_{j}^{i}$

Putting value of $A_{i}^{j}=A_{j}^{i}$ in ((9)), we get 
$W_{i, j}=\frac{1}{\pi r^{2}}\left\{\frac{r^{2} \cos ^{-1}\left(\frac{d_{i, j}}{2 r}\right)}{2}-\frac{h_{i, j} d_{i j}}{4}+\frac{r^{2} \cos ^{-1}\left(\frac{d_{i, j}}{2 r}\right)}{2}-\right.$

$\left.\frac{h_{i, j} d_{i j}}{4}\right\}$

Now putting values of $h_{i, j}$ in ((11)), we get [25]:

$$
\begin{aligned}
w_{i, j} & =\frac{1}{\pi} \cos ^{-1}\left(\frac{d_{i, j}}{2 r}\right)-\frac{d_{i j}}{2 \pi r^{2}} \sqrt{r^{2}-\frac{d_{i j}^{2}}{4}} \\
& =\frac{1}{\pi} \cos ^{-1}\left(\frac{d_{i, j}}{2 r}\right)-\frac{d_{i j}}{4 \pi r^{2}} \sqrt{(2 r)^{2}-d_{i j}^{2}} \\
& =\frac{1}{\pi} \cos ^{-1}\left(\frac{d_{i, j}}{2 r}\right)-\frac{d_{i j}}{\pi(2 r)^{2}} \sqrt{(2 r)^{2}-d_{i j}^{2}}
\end{aligned}
$$

By put $\theta=2 r$, we get

$w_{i, j}=\frac{1}{\pi} \cos ^{-1}\left(\frac{d_{i, j}}{\theta}\right)-\frac{d_{i j}}{\pi \theta^{2}} \sqrt{\theta^{2}-d_{i j}^{2}}$

We see that parameter $\theta$ controls the correlation among active sensors and $\mathrm{w}_{\mathrm{i}, \mathrm{j}}$ can be rewritten in general form as follows [25]

$\mathrm{w}_{\mathrm{i}, \mathrm{j}}=$

$\left(\begin{array}{ll}\frac{1}{\pi} \cos ^{-1}\left(\frac{d_{i, j}}{\theta}\right)-\frac{d_{i j}}{\pi \theta^{2}} \sqrt{\theta^{2}-d_{i j}^{2}}, & \text { for } 0 \leq d_{i, j} \leq \theta, \\ 0, & \text { ford }_{i, j}>\theta,\end{array}\right.$

where $r$ is considered to be fixed as specified sensing range of a given sensor. We consider that all sensors have similar sensing range. The proposed two stage algorithm is summarized in Table 1.

\subsection{Performance Analysis}

As under hypothesis $\mathcal{H}_{0}, \quad \mathbf{x}_{\mathrm{LE}_{\mathrm{E}}} \sim \mathcal{N}\left(0, \sigma_{\mathrm{V}}^{2} \mathbf{I}_{\mathrm{L}_{\mathrm{E}}}\right)$ and under hypothesis $\mathcal{H}_{1}, \mathbf{x}_{\mathrm{L}} \sim \mathcal{N}\left(\mathbf{P}_{\mathrm{L}_{\mathrm{E}}}, \sigma_{\mathrm{v}}^{2} \mathbf{I}_{\mathrm{LE}}\right)$, then [16, 17]

$\mathbf{x}_{\mathrm{LE}_{\mathrm{E}}}^{\mathrm{T}} \mathbf{W} \mathbf{x}_{\mathrm{LE}_{\mathrm{E}}} \sim\left(\begin{array}{ll}\chi_{\mathrm{L}_{\mathrm{E}}}^{2}(\lambda) & \text { for } \mathcal{H}_{1} \\ \chi_{\mathrm{L}_{\mathrm{E}}}^{2} & \text { for } \mathcal{H}_{0}\end{array}\right.$

where $\lambda=\mathbf{P}_{\mathrm{L}_{\mathrm{E}}}^{\mathrm{T}} \mathbf{W} \mathbf{P}_{\mathrm{L}_{\mathrm{E}}}$ and $\mathrm{L}_{\mathrm{E}}=\mathrm{E}[\mathrm{L}]$ is the estimated value of L. Keeping this discussion into consideration, the detection probability becomes

$\mathrm{P}_{\mathrm{D}}\left(\mathrm{L}_{\mathrm{E}}\right)=\mathrm{Q}_{\mathrm{L}_{\mathrm{E}}^{2}(\lambda)}\left(\gamma_{\mathrm{F}}\right)$ and probability of false alarm is

$\mathrm{P}_{\mathrm{FA}}\left(\mathrm{L}_{\mathrm{E}}\right)=\mathrm{Q}_{\chi_{\mathrm{L}_{\mathrm{E}}^{2}}}\left(\gamma_{\mathrm{F}}\right)$

We can observe that the detection performance depends on $L$. This can be found empirically with histograms, as histogram can be used to find the estimated distribution of $\mathrm{L}, \mathrm{P}_{\mathrm{L}}(\mathrm{l})$. With $\mathrm{P}_{\mathrm{L}}(\mathrm{l})$ the expression of average detection probability $\overline{\mathrm{P}}_{\mathrm{D}}$ are given as $[16,17]$ :

$\overline{\mathrm{P}}_{\mathrm{D}}=\mathrm{E}_{\mathrm{LE}_{\mathrm{E}}}\left[\mathrm{P}_{\mathrm{D}}\left(\mathrm{L}_{\mathrm{E}}\right)\right]=\sum_{\mathrm{l}=1}^{\mathrm{L}_{\max }} \mathrm{P}_{\mathrm{D}}(\mathrm{l}) \mathrm{f}_{\mathrm{LE}_{\mathrm{E}}}(\mathrm{l})$

Similarly, for the average probability of false alarm, $\overline{\mathrm{P}}_{\mathrm{FA}}$

$\overline{\mathrm{P}}_{\mathrm{FA}}=\mathrm{E}_{\mathrm{L}_{\mathrm{E}}}\left[\mathrm{P}_{\mathrm{FA}}\left(\mathrm{L}_{\mathrm{E}}\right)\right]=\sum_{\mathrm{l}=1}^{\mathrm{L}_{\max }} \mathrm{P}_{\mathrm{FA}}(\mathrm{l}) \mathrm{f}_{\mathrm{L}_{\mathrm{E}}}(\mathrm{l})$

The performance of detection for a particular detector is expressend in terms of $\overline{\mathrm{P}}_{\mathrm{D}}$ and $\overline{\mathrm{P}}_{\mathrm{FA}}$.

Table 1: Summary of the proposed two stage detection mechanism

- In response to queries received from the fusion center, each sensor in WSN takes N observations to perform the local detection process using GLRT test given in ((3)).

- Each sensor decides in favor of either $\mathcal{H}_{1}$ or $\mathcal{H}_{0}$.

- Sensors with decision $\mathcal{H}_{1}$ are classified as active sensors and sensors with decision $\mathcal{H}_{0}$ are classified as non-active sensors.

- Only the active sensors send their information (i.e., $\overline{\mathrm{y}}_{\mathrm{i}}, \mathrm{i}=1,2, \ldots, \mathrm{L}$ ) to the fusion center and non-active sensors will switch back to inactive mode, hence, local censoring is achieved.

- Upon receiving the information, the fusion center exploits location information of $\mathrm{L}$ active sensors and $\mathbf{x}=\left[\overline{\mathrm{y}}_{1}, \overline{\mathrm{y}}_{2}, \ldots, \overline{\mathrm{y}}_{\mathrm{L}}\right]$ to perform the detection process given in $((5))$.

\section{RESULTS AND DISCUSSION}

To evaluate the detection performance of the proposed scheme, numerical simulations are presented in this section. For these simulations we have used a sensor network of total 256 sensor nodes that are deployed in the form of a grid in a square area as shown in Fig 4. 
We consider a target that is located at the center of the field and emitting power $\mathrm{P}_{0}=2 \mathrm{~dB}$. As it is argued in earlier sections, this power is assumed to be received by a subset of sensors present in the event region as shown in Fig. 4. Noise power at each sensor is considered to be $\sigma=2 \mathrm{~dB}$. We further assume that a noise power added by the fusion center is $\sigma=-5 \mathrm{~dB}$. Rest of the parameters are defined during each experiment performed.

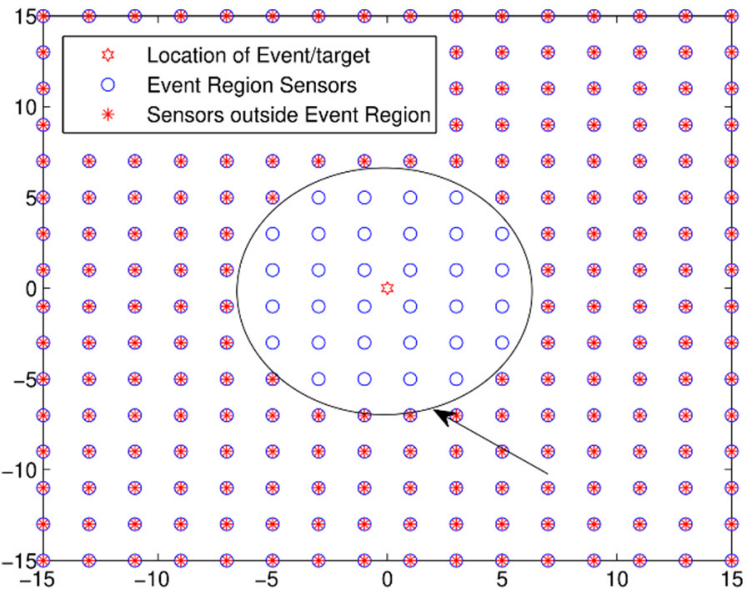

Fig. 4: Sensor deployment and event region based on the range of event signal

With the aforementioned parameters the scenerio of the hypothesis $\mathcal{H}_{0}$ is demonstrated in Fig. 5, where we can see that although event signal is not present but multiple sensors declare $\mathcal{H}_{1}$ falsely. Such erroneous declarations from the sensors are due to noise. However, we can observe that these false active sensors are not in the form of cluster rather they are randomly located in the field. Similarly, $\mathcal{H}_{1}$ is demonstrated in Fig. 6, where we can observe that multiple sensors declare $\mathcal{H}_{1}$ are in the form of cluster. This show that in addition to signal energy, the cluster information can also be used as an additional information.

In order to demonstrate the effectiveness of our proposed scheme, we use the Reciever Operating Curve (ROC) to obtain the Probability of detection vs Probability of False alarm using algorithm given in [26]. These results are shown in Fig. 7, where we can see that the proposed scheme out performs the traditional GLRT scheme. We remark that the GLRT is asymptotically optimal detection test.

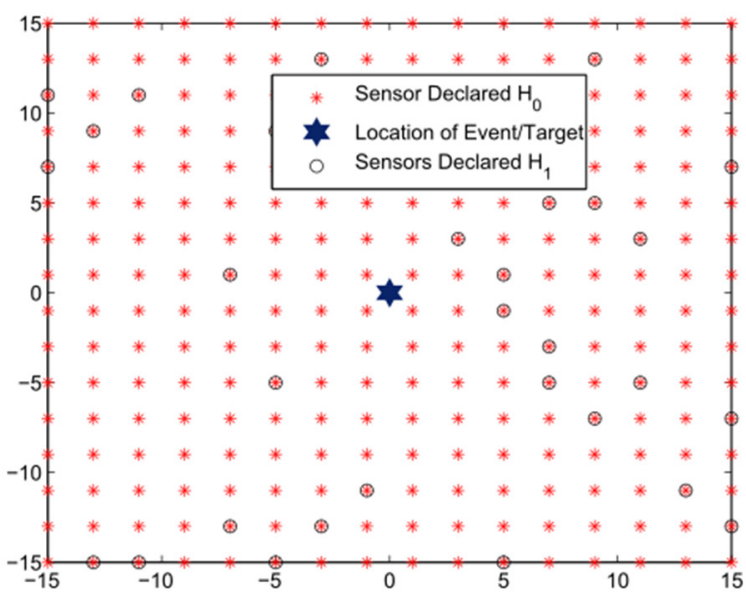

Fig. 5: Demonstration of Hypothesis $\mathcal{H}_{0}$

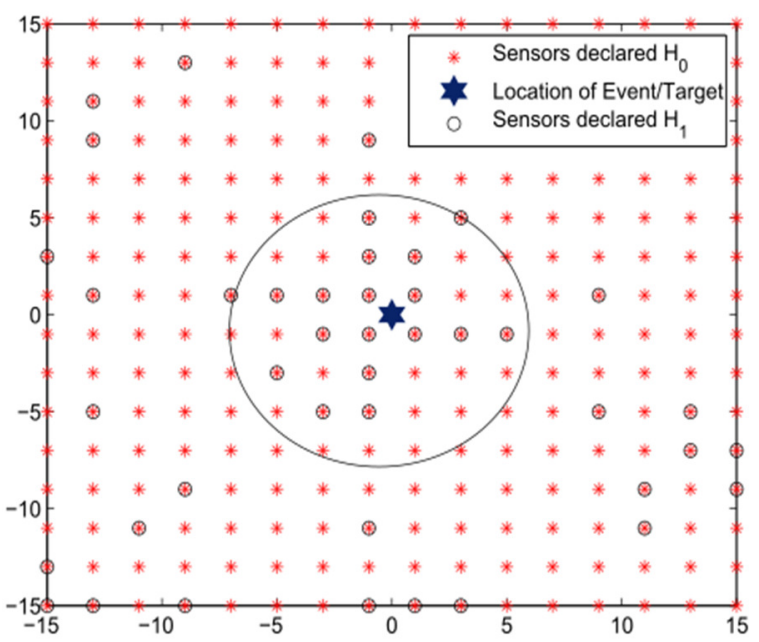

Fig. 6: Demonstration of Hypothesis $\mathcal{H}_{1}$

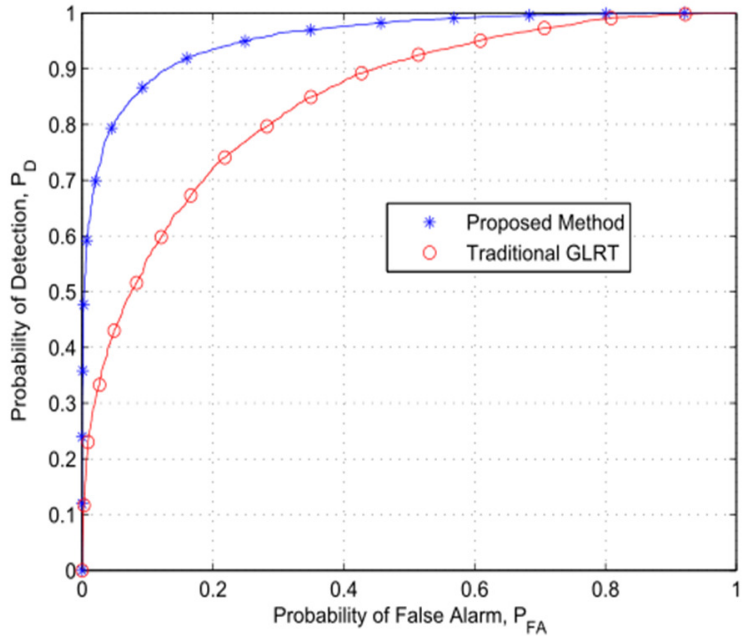

Fig. 7: Probability of Detection vs Probability of False Alarm 


\section{CONCLUSION}

We have proposed a novel detection scheme for wireless sensor networks that exploits the fact that sensors located near to the event region have more credible information, whereas the reports of the rest of the far away sensors will be prone to faulty detection. In order to exploit this fact, a two-stage detection mechanism is considered that exploits the spatial proximity of the sensors present inside the event region. In the proposed mechanism, first of all each sensor in the field makes its own local decision. If the local decision is in favor of hypothesis $\mathcal{H}_{1}$, it sends observations to fusion center otherwise remains silent, hence, local censoring is achieved. Upon receiving the local observations of selected sensors, the fusion center combines the known location of these sensors with the received observations to draw the final decision. Simulation results have been provided to assess the performance of the proposed scheme that show the proposed scheme has superior performance over the traditional detection scheme.

\section{ACKNOWLEDGMENT}

This paper and the research behind it would not have been possible without the exceptional support of Department of Electrical Engineering, University of Engineering and Technology, Peshawar.

\section{REFERENCES}

1. Tanenbaum A.S., Gamage C., Crispo B., "Taking sensor networks from the lab to the jungle", IEEE Computer, Vol. 39, No. 8, pp. 98-100, 2006.

2. Chamberland J.-F., Veeravalli V.V., "Wireless sensors in distributed detection applications", IEEE Signal Processing Magazine, Vol. 24, pp.16-25, 2007.

3. Culler D., Estrin D., Srivastava M., "Overview of sensor networks", IEEE Computer, Vol. 37, No.8, pp. 41-49, August 2004.

4. Manges W.W., "Wireless sensor network topologies", Sensors Magazine, Vol. 17, No. 5, May 2000.

5. Akyildiz I., Su W., Sankarasubramaniam Y., Cayirci E.. "A survey on sensor networks", IEEE Communications Magazine, Vol. 40, No. 8, pp. 102-114, August 2002.

6. Guerriero M., Willett P., Glaz J., "Distributed target detection in sensor networks using scan statistics", IEEE Transactions on Signal Processing, Vol. 57, No.7, pp. 2629-2639, 2009.

7. Gupta K., Merchant S.N., Desai U.B., "A novel multistage decision fusion for cognitive sensor networks using and and or rules", Digital Signal Processing, Vol. 42, No. C, pp. 27 - 34, 2015.

8. Fang J., Li H., "Distributed event region detection in wireless sensor networks", EURASIP Journal on Advances in Signal Processing, Vol. 2008, November 2007.

9. Akyildiz I. F., Vuran M. C., Akan O. B., "On exploiting spatial and temporal correlation in wireless sensor networks", Proceedings of the $2^{\text {nd }}$ International Conference on Modeling and Optimization in Mobile, Ad Hoc and Wireless Networks (WiOpt), pp. 71-80, Cambridge, U.K., 2004.

10. Zhang Y., Meratnia N., Havinga P., "Outlier Detection Techniques for Wireless Sensor Networks: A Survey", IEEE Communications Surveys \& Tutorials, Vol.12, No. 2, 2010.

11. Vankayalapati N., Kay S. M., "Asymptotically optimal detection/ localization of LPI signals of emitters using distributed sensors", Proc. of the SPIE, 7706:77060U-77060U-9, 2010.

12. Anandkumar A., Tong L., Swami A., "Detection of Gauss-Markov random fields with nearestneighbor dependency", IEEE Transactions on Information Theory, Vol. 55, pp. 816 - 827, 2009.

13. Hong C.-O., Choi Y.-H.. "Proximity-based robust event detection in wireless sensor networks", International Journal of Distributed Sensor Networks, Vol. 2014, 2014.

14. H.H.W.J., Giovanni Iacca, Arturo Tejada, Heinrich J.Wörtche, and Antonio Liotta, "Spatial anomaly detection in sensor networks using neighborhood information", Information Fusion, Vol. 33, pp.41 - 56, 2017.

15. Xu C., Kay S. M., "On centralized composite detection with distributed sensors", In Proc. of the IEEE Radar Conference, pages 1-6, 2008.

16. Ali S., Seco-Granados G., Lopez-Salcedo. J.A., "Spectrum sensing with spatial signatures in the presence of noise uncertainty and shadowing", EURASIP Journal on Wireless Communications 
and Networking, Vol. 2013, June 2013.

17. Ali S., López-Salcedo J.A., Seco-Granados G., "Improved GLRT based on the exploitation of spatial correlation between neighbouring sensors", In Proc. of 19th EUSIPCO, pp. 10451049, 2011.

18. Ding F., Song G., Yin K., Li J., Song A., “A GPSenabled wireless sensor network for monitoring radioactive materials", Sensors and Actuators A: Physical, Vol. 155, No. 1, pp. 210 - 215, 2009.

19. Buchli B., Sutton F., Beutel J., "GPS-Equipped Wireless Sensor Network Node for HighAccuracy Positioning Applications", pp. 179195, Springer Berlin Heidelberg, 2012.

20. Niu R., Varshney P.K., "Decision fusion in a wireless sensor network with a random number of sensors", In IEEE International Conference on Acoustics, Speech, and Signal Processing (ICASSP), Vol. 4, pp. 861-864, March 2005.

21. Li D., Wong K. D., Hu Y.H., Sayeed A. M., "Detection, classification, and tracking of targets" IEEE Signal Processing Magazine, Vol. 19, No. 2, pp. 17-29, March 2002.

22. Kay S. M., "Fundamentals of Statistical Signal Processing”, Vol. 2, Detection Theory. Prentice Hall, 1998.

23. Yessembayev A., Sarkar D., Sikder F., "Detection of good and bad sensor-nodes in presence of malicious attacks, and its application to data aggregation”, IEEE Transactions on Signal and Information Processing over Networks, pp. 549563, 2018.

24. Das M., Ghosh S. K., "Measuring Moran's-I in a cost-efficient manner to describe a land-cover change pattern in large-scale remote sensing imagery", IEEE Journal of Selected Topics in Applied Earth Observations and Remote Sensing, Vol. 10, No. 6, pp. 2631-2639, June 2017.

25. Shakya R. K., Singh Y. N., Verma N. K., "A novel spatial correlation model for wireless sensor network applications", In Ninth International Conference on Wireless and Optical Communications Networks (WOCN), pp. 1-6, Sept 2012.

26. Fawcett T., "ROC graphs: Notes and practical considerations for researchers", Technical Report HPL-2003-4, HP Laboratories, 2003. 